\title{
Cytochrome P450 Metabolites of Arachidonic Acid Are Potent Inhibitors of Vasopressin Action on Rabbit Cortical Collecting Duct
}

Darrell L. Hirt, Jorge Capdevila, J. R. Falck, ${ }^{\star}$ Matthew D. Breyer, and Harry R. Jacobson

Division of Nephrology, Department of Medicine, Veterans Administration Medical Center and Vanderbilt University, Nashville, Tennessee 37232; and *Department of Molecular Genetics, University of Texas

Southwestern Medical Center at Dallas, Dallas, Texas 75235

\begin{abstract}
AA is metabolized by a cytochrome P450, NADPH-dependent epoxygenase to four regioisomeric epoxyeicosatrienoic acids (EETs). The EETs are further hydrated enzymatically to their respective diols, vic-dihydroxyeicosatrienoic acids (DHETs). We studied the effect of pretreatment with DHETs on 10 $\mu \mathrm{U} / \mathrm{cm}^{2}$ arginine vasopressin (AVP)-stimulated hydraulic conductivity $\left(L_{\mathrm{p}}\right)\left(L_{\mathrm{p}} \times 10^{-7} \mathrm{~cm} / \mathrm{atm} / \mathrm{s}\right.$, mean $\left.\pm \mathrm{SE}\right)$ in rabbit cortical collecting ducts (CCDs) perfused in vitro at $37^{\circ} \mathrm{C}$. At $10^{-6} \mathrm{M}$ all four DHETs were potent inhibitors of the hydroosmotic effect of AVP. 14,15-DHET was the most potent isomer; it reduced AVP-induced $L_{p}$ from a control value of 234.75 $\pm 11.7, n=17$, to a value of $95.2 \pm 8.39, n=5, P$ $<0.0001$, a reduction of AVP-mediated water flow of $60 \%$. The inhibitory effect of 14,15-DHET was dose dependent and significant to nanomolar concentrations. 14,15-DHET at $10^{-7}$ $M$ was as potent an inhibitor of AVP's activity as was $10^{-7} \mathrm{M}$ PGE $_{2}$. AVP's hydroosmotic effect is mediated through its intracellular second messenger, cAMP. 8-p-ChlorophenylthiocAMP (CcAMP) at $10^{-4} \mathrm{M}$ induced a peak $L_{\mathrm{p}}$ of 189.6 \pm 11.0 , $n=8$; pretreatment with $10^{-6} \mathrm{M} 14,15-D H E T$ reduced CcAMP-peak $L_{\mathrm{p}}$ to $132.0 \pm 13.4, n=5, P<0.01$, demonstrating a post-cAMP effect. Gas chromatography/mass spectroscopy suggests that EETs are present in extracts purified from CCDs. We conclude that cytochrome $\mathbf{P 4 5 0}$ epoxygenase eicosanoids are potent inhibitors of the hydroosmotic effect of vasopressin and are endogenous constituents of normal CCDs, the major target tissue for AVP.
\end{abstract}

\section{Introduction}

Systematic study of the oxygenated metabolism of AA in health and disease has documented the importance of its metabolites to renal physiology and pathophysiology. Eicosanoids have been implicated in the renal response to peptide hormones and other cellular messengers. They affect renal circulation, contribute to inflammatory processes within the kid-

Preliminary reports of work cited in this manuscript were presented at the 1987 National Meeting of the AAP, ASCI, and AFCR, San Diego, $\mathrm{CA}$; at the Xth International Congress of Nephrology, London; and at the 1987 Annual Meeting of the American Society of Nephrology, Washington, DC.

Address correspondence to Dr. Darrell Hirt, Room S3223 Medical Center North, Vanderbilt University, Nashville, TN 37232.

Received for publication 29 December 1987 and in revised form 7 August 1989.

J. Clin. Invest.

(c) The American Society for Clinical Investigation, Inc.

$0021-9738 / 89 / 12 / 1805 / 08 \quad \$ 2.00$

Volume 84, December 1989, 1805-1812 ney, and have important effects on solute and water transport (1-4).

AA can be metabolized by three distinct enzymatic pathways: $(a)$ by cyclooxygenase to form biologically active PGs and TXs; $(b)$ by lipoxygenases to form hydroperoxy fatty acids, monohydroxy fatty acids, and leukotrienes; and $(c)$ by a recently described cytochrome P450 NADPH-dependent epoxygenase $(5-10)$. This third pathway of AA metabolism has been documented in the kidney $(5,7,8)$. Epoxidation of each of the double bonds of AA generates four regioisomeric epoxides: the 5,6-, 8,9-, 11,12-, and 14,15-epoxyeicosatrienoic acids (EETs). ${ }^{1}$ The epoxides can be hydrated by cytosolic epoxide hydratases to form the corresponding diols: 5,6-, 8,9-, 11,12-, and 14-15-dihydroxyeicosatrienoic acids (DHETs) (Fig. 1).

The presence of a cytochrome P450 NADPH-dependent monoxygenase in numerous tissues including the kidney has been documented and potentially important biologic actions of P450 eicosanoids in several physiologic systems have been observed (10-18). 5,6-EET has been shown to suppress sodium absorption and potassium secretion in microperfused rabbit cortical collecting ducts (CCDs) (11). In a cell preparation from medullary thick ascending limb of Henle, the production of metabolites of this pathway is altered by drugs, hormones, and maneuvers that result in experimental hypertension (16). NaK ATPase activity in thick-limb cells is reduced by these products $(12,16)$. Finally, recent studies on toad urinary bladders have shown that EETs and DHETs inhibit vasopressin-induced osmotic water flow (18).

We report here studies that examined the effect of diols (DHETs) of renal P450 epoxygenase on the hydroosmotic response of the rabbit CCD to arginine vasopressin (AVP) and the cell permeant, phosphodiesterase-resistant cAMP analogue, 8-p-chlorophenylthio-cAMP (CcAMP).

\section{Methods}

Experiments were performed using the technique of in vitro microperfusion of isolated nephron segments as originally described by Burg et al. (19). Female New Zealand white rabbits were maintained on ad lib tap water and chow. They were killed by decapitation and the left kidney was rapidly exposed and removed. $1-\mathrm{mm}$-thick slices of kidney were made and these were placed in ice-chilled medium that had the same composition as the bath solution described below. A slice was transferred in the same chilled medium to a dissecting microscope where individual CCDs were dissected freehand with sharpened forceps. An isolated CCD (1-2.5 mm long) was then transferred to a thermostatically controlled lucite bath chamber on an inverted micro-

1. Abbreviations used in this paper: CcAMP, 8-p-chlorophenylthiocAMP; CCD, cortical collecting duct; DHET, dihydroxyeicosatrienoic acid; EET, epoxyeicosatrienoic acid; $L_{\mathrm{p}}$, hydraulic conductivity; $m / z$, mass/ionic charge ratio; NICI GC/MS, negative ion chemical ion gas chromatography/mass spectroscopy; PFB, pentaflourobenzyl; $\boldsymbol{R}_{\mathrm{t}}$, retention time. 


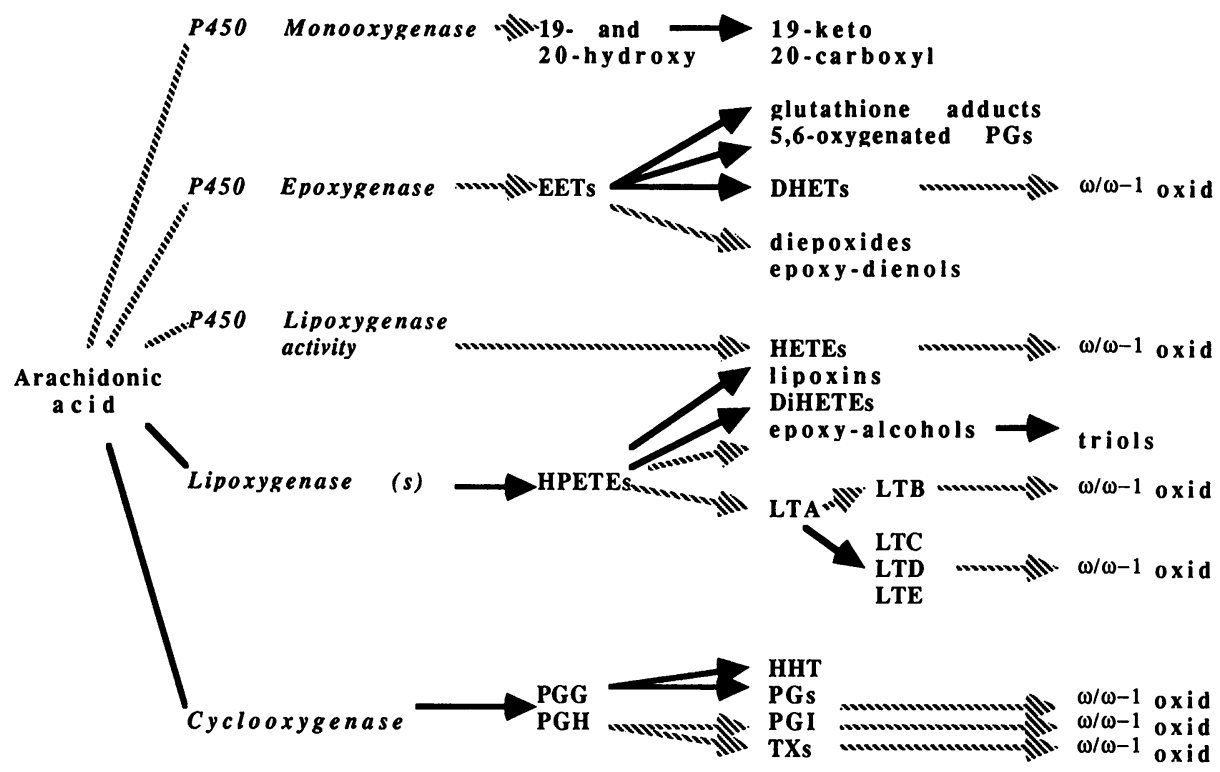

Figure 1. Potential or known metabolic pathways of AA metabolism. Established and/or suspected pathways catalized by cytochrome $\mathbf{P 4 5 0}$ or related enzymes are indicated by dashed lines. Pathways involving cyclooxygenase, lipoxygenases, or other enzymes are shown by the solid lines. HPETE/ HETE, Hydroperoxy/hydroxyeicosatetraenoic acid; $L T$, leukotriene; $H H T$, hydroxyheptadecatrienoic acid; $P G I$, prostacyclin.

scope stage. Each end of the tubule was gently sucked into micropipettes and it was perfused using a concentric micropipette system. Generally CCDs were perfused within 30 min of killing the animal.

After cannulation of a tubule, bath was continuously exchanged at a flow rate of $0.5 \mathrm{~cm}^{2} / \mathrm{min}$ with an infusion pump (Sage Instruments, Cambridge, MA). Transepithelial voltage was monitored using an electrometer (model 602; Keithley Instruments, Inc., Cleveland, $\mathrm{OH}$ ) and recorded on a strip chart recorder. The bath was warmed to $37-38^{\circ} \mathrm{C}$. Perfusion rate was adjusted to $12-20 \mathrm{nl} / \mathrm{min}$ by adjustment of hydrostatic pressure to the perfusion pipette. These flow rates were used to maintain a transepithelial osmotic gradient over the length of the tubule. Perfused fluid was collected in a constriction pipette of known volume (70-100 $\mathrm{nl})$.

\section{Solutions}

Composition of dissection medium and bath were as follows (in millimolar): $\mathrm{NaCl} 105, \mathrm{NaHCO}_{3} 25, \mathrm{NaOAc} 10, \mathrm{NaHPO}_{4} 2.3, \mathrm{KCl} 5, \mathrm{CaCl}_{2}$ $1.8, \mathrm{MgSO}_{4} 1$, glucose 8.3 , alanine 5 , and BSA $1 \mathrm{mg} \%$ (osmolality, 300 mosM). Isotonic perfusate was the same as above with the exception of the serum albumin. Hypotonic perfusate (150 mosM) was also the same except for reduction in $\mathrm{NaCl}$ content to $30 \mathrm{mM}$ and the addition of tritium-labeled inulin as a volume marker. Perfusates, bath, and dissection media were gassed to equilibrium with $95 \% \mathrm{O}_{2} / 5 \% \mathrm{CO}_{2}$ to pH 7.40 and drawn anaerobically into syringes.

The four regioisomeric EETs and DHETs were synthesized either from $\left[1-{ }^{14} \mathrm{C}\right] \mathrm{AA}(0.010 \mu \mathrm{Ci} / \mu \mathrm{mol})$ or $\left[5,6,8,9,11,12,14,15-{ }^{2} \mathrm{H}\right] \mathrm{AA}(\mathrm{a}$ gift of the Upjohn Co., Kalamazoo, MI) according to previously described methods (20-22). The synthetic compounds were purified by reverse-phase HPLC and quantified by liquid scintillation. Stock solutions in ethanol were kept under argon gas at $-70^{\circ} \mathrm{C}$ until use. DHET stocks were changed weekly. Prior assays performed by us had determined that the DHETs were stable under these conditions.

$\mathrm{PGE}_{2}$ was also reconstituted in ethanol to obtain a stock solution that was handled the same as for DHETs. The sodium salt of CCAMP was dissolved in an aqueous solution, divided into aliquots, and stored at $-20^{\circ} \mathrm{C}$ until thawed before use. A stock of AVP was made with acidified Ringers bicarbonate ( $\mathrm{pH} 4.0$ ) and stored at $4^{\circ} \mathrm{C}$. $10 \%$ collagenase in Krebs-Ringer solution was used to flush in situ, via the renal artery, kidneys dissected for CCDs subjected to the lipid extraction and negative ion chemical ion gas chromatography/mass spectroscopy (NICI GC/MS) analysis.

\section{Calculations}

Net volume flux $\left(J_{\mathrm{v}}\right.$; nanoliters/minute per millimeter) was calculated for each collection using the equation $J_{\mathrm{v}}=\left(V_{\mathrm{i}}-V_{\mathrm{o}}\right) / L$, where $V_{\mathrm{o}}$ and
$V_{\mathrm{i}}$ are the collection and perfusion rates (nanoliters/minute), respectively, and $L$ is the tubule length measured directly using an eyepiece reticle. $V_{\mathrm{o}}$ is determined from the equation $V_{\mathrm{o}}=V_{\mathrm{p}} / t$, in which $V_{\mathrm{p}}$ is the volume of the constriction pipette (nanoliters) and $t$ is the collection time (minutes). $V_{\mathrm{i}}=V_{\mathrm{o}} \times C_{\mathrm{o}} / C_{\mathrm{i}}$, in which $C_{\mathrm{o}}$ and $C_{\mathrm{i}}$ are the radioactivity of tritium-labeled inulin (counts per minute per nanoliter) in collected fluid and perfusate, respectively, counted by liquid scintillation counter (model 6892; Tracor Analytic Inc., Elk Grove Village, IL).

Hydraulic conductivity ( $L_{\mathrm{p}}$; centimeters/atmosphere per second) was calculated according to the equation of Du Bois et al. (23):

$$
\begin{aligned}
& (1 / \mathrm{RTS}) \cdot\left(1 / O_{\mathrm{b}}\right)^{2} \cdot\left\{O_{\mathrm{b}} \cdot\left(V_{\mathrm{i}}-V_{\mathrm{o}}\right)+O_{\mathrm{i}} \cdot V_{\mathrm{i}}\right. \\
& \left.\times L_{\mathrm{n}}\left[\left(O_{\mathrm{b}}-O_{\mathrm{i}}\right) \cdot V_{\mathrm{i}} /\left(O_{\mathrm{b}} \cdot V_{\mathrm{o}}-O_{\mathrm{i}} \cdot V_{\mathrm{i}}\right)\right]\right\},
\end{aligned}
$$

where $\mathrm{R}$ is the gas constant, $T$ is the temperature of the bath (degrees Kelvin), $S$ is the luminal surface area of the tubule calculated using an assumed luminal diameter of $20 \mu \mathrm{m}$, and $O_{\mathrm{b}}$ and $O_{\mathrm{i}}$ are the osmolalities of bath medium and perfusate, respectively.

\section{Experimental protocols}

Incubation of cannulated tubules was performed for 30-45 min at $37^{\circ} \mathrm{C}$ using isotonic perfusate. During this time cell morphology was observed for damage and perfusion flow rate was adjusted. Transepithelial potential difference was measured. Subsequently, isotonic perfusate was changed to hypotonic $\left[{ }^{3} \mathrm{H}\right]$ inulin-containing perfusate.

Effect of DHETs on AVP response. After perfusate exchange the bath was changed to one containing vehicle alone (ethanol; final concentration in bath, $0.001 \%$ ) or one containing DHET in vehicle to achieve a concentration in the bath of $10^{-6} \mathrm{M}$. Tubules were incubated for an additional $30 \mathrm{~min}$ before three collections were obtained to determine $J_{\mathrm{v}}$ and $L_{\mathrm{p}}$ values in basal conditions for vehicle and DHET. Next, the bath was changed to one containing vehicle or $10^{-6} \mathrm{M}$ DHET and $10 \mu \mathrm{U} / \mathrm{cm}^{2}$ AVP. This submaximal dose of AVP was chosen to allow us to observe either stimulatory or suppressive effects of DHET on the hydroosmotic effect of AVP. After AVP exposure serial collections were obtained to determine peak $J_{\mathrm{v}}$ and $L_{\mathrm{p}}$ response. Peak response was defined as the mean of three collections whose $J_{v} / L_{\mathrm{p}}$ had the highest values on AVP exposure. Generally, the peak response occurred within 20 to 40 min of bath exchange. The effect of pretreatment of CCDs with each of the four isomeric DHETs was thus determined.

Dose response of 14,15-DHET. Having observed that 14,15-DHET pretreatment caused the most potent inhibition of the hydroosmotic effect of AVP, we established a dose-response relationship for this compound. Radiolabeled $\left[{ }^{14} \mathrm{C}\right] 14,15-\mathrm{DHET}$ was measured and ap- 
propriate dilutions were made to obtain stock solutions such that the volume of ethanol vehicle added to the bath was the same for all experiments. Bath exchanges and collections for control and experimental conditions were performed according to the time course and methods described in the previous protocol.

Comparison of 14,15-DHET vs. PGE on the AVP response. In these experiments we compared the effect of pretreatment with equimolar concentrations $\left(10^{-7} \mathrm{M}\right)$ of $14,15-\mathrm{DHET}$ and $\mathrm{PGE}_{2}$ on the response of CCDs to $10 \mu \mathrm{U} / \mathrm{cm}^{2}$ AVP. Other than the concentrations of each arachidonate metabolite that was present in the bath, the protocols followed were the same as above.

Effect of 14,15-DHET on CCAMP response. As initial studies to determine the mechanism by which DHETs were inhibiting the AVP response, we also examined whether 14,15-DHET would affect the hydroosmotic response of CCDs to the intracellular second messenger of vasopressin, cAMP. Tubules were perfused and bathed as described, then pretreated with either vehicle or $10^{-6} \mathrm{M} 14,15$-DHET, and basal collections were made for $J_{\mathrm{v}}$ and $L_{\mathrm{p}}$. Subsequently, the bath was exchanged to one containing vehicle or DHET and $10^{-4} \mathrm{M}$ CcAMP and peak response was measured. In CcAMP experiments the peak response was defined as above and occurred within 20 to $50 \mathrm{~min}$ after exposure to CcAMP.

Analysis of harvested CCDs for epoxygenase metabolites (Fig. 2). Rabbit CCDs were freehand dissected from collagenase perfused kidney slices in ice-chilled dissection medium using a dissection microscope as described above. Care was taken to remove adhering thick limbs from the individual CCDs. The lengths of the tubules were measured by eyepiece reticle. Dissection was completed within $60 \mathrm{~min}$ after death. Harvested tubules were transferred with minimal dissection medium to glass test tubes containing ice-chilled HPLC reagent grade methanol.

The CCDs were homogenized in ice-cold $0.2 \mathrm{M} \mathrm{KCl}$ containing $40 \% \mathrm{CH}_{3} \mathrm{OH}$ and $0.01 \%$ BHT (wt/vol). After adding $0.02 \mu \mathrm{Ci}$ of $\left[5,6,8,9,11,12,14,15-{ }^{3} \mathrm{H}\right] 14,15$-EET $(150-170 \mathrm{Ci} / \mathrm{mmol})$ in ethanol as recovery standard, an equal volume of cold $\mathrm{CHCl}_{3}$ was added with vigorous mixing. After phase separation the aqueous layer was extracted with 2 vol of $\mathrm{CHCl}_{3}-\mathrm{CH}_{3} \mathrm{OH}(2: 1)$.

The combined organic extracts were purified as described (24). Briefly, the lipid extract was hydrolyzed in the presence of $0.2 \mathrm{M} \mathrm{KOH}$, $80 \% \mathrm{CH}_{3} \mathrm{OH}$ for $60 \mathrm{~min}$ at $40^{\circ} \mathrm{C}$ under an argon blanket. After neutralization and extraction into ethyl acetate the samples were purified by reverse-phase HPLC as described (25). HPLC fractions with retention times corresponding to those of synthetic EETs were collected, evapo-

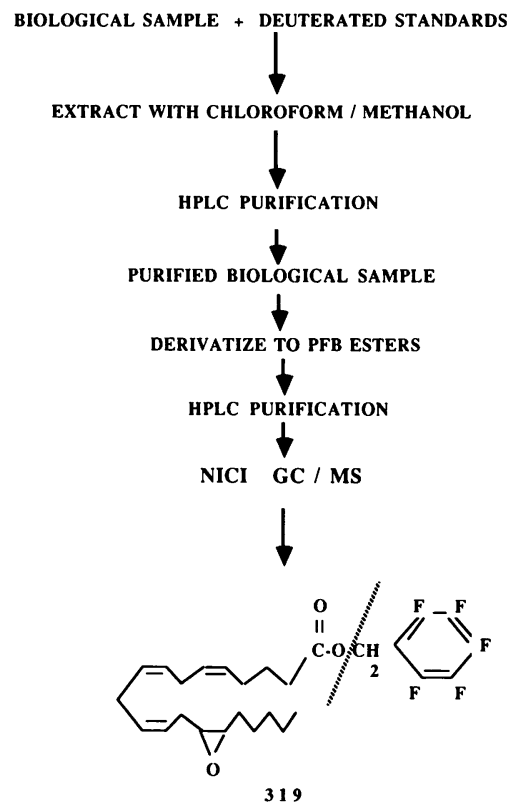

Figure 2. Scheme of methodology used to identify presence of cytochrome $\mathbf{P 4 5 0}$ NADPH-dependent EETs in biological samples. EETs are identified by analysis of GC retention times using selective ion monitoring during NICI GC/MS. After cleavage the EETPFB esters share a common ion fragment at $m / z 319$ under NICI conditions. rated under argon, and derivatized to the corresponding pentaflourobenzyl (PFB) derivatives. The EET-PFB esters were purified by reverse-phase HPLC as described (25). Samples were analyzed by NICI GC/MS on a Nermag quadrapole instrument (model R1010C) interfaced to a gas chromatograph (Varian Associates, Palo Alto, CA) using a 30-m SPB5 fused silica capillary column $(0.32 \mathrm{~mm}$ i.d., $0.25-\mu \mathrm{m}$ coating thickness; Supelco, Inc., Bellefonte, PA) with helium and methane as carrier and reagent gases, respectively. Instrumental conditions were as follows: analyzer pressure, $6.4 \times 10^{-6}$ Torr; source temperature, $200^{\circ} \mathrm{C}$; electron energy, $90 \mathrm{eV}$; dynode, $+5 \mathrm{kV}$. For analysis, the GC column temperature was programmed from 100 to $310^{\circ} \mathrm{C}$ at $20^{\circ} \mathrm{C} / \mathrm{min}$ and held at $310^{\circ} \mathrm{C}$ for $10 \mathrm{~min}$. Injections were made in hexane.

\section{Reagents}

AA was from NuChek Prep, Elysian, MN. Radiolabeled AA was from Amersham Corp., Arlington Heights, IL. AVP, PGE $_{2}$, and CcAMP were purchased from Sigma Chemical Co., St. Louis, MO. All solvents were reagent grade. Radioactive tritium-labeled inulin was purchased from DuPont NEN Products Co., Boston, MA.

\section{Statistics}

Data are presented as mean $\pm \mathrm{SE}$; statistical comparisons were made using one-way analysis of variance. A difference of $P<0.05$ was considered statistically significant.

\section{Results}

As $J_{\mathrm{v}}$ and $L_{\mathrm{p}}$ are qualitatively the same as a parameter of water transport, only $L_{\mathrm{p}} \mathrm{s}$ are presented below.

Effect of DHETs on AVP response (Fig. 3). Equimolar concentrations of each DHET were used to assess their effect on AVP-stimulated osmotic water flow in CCDs. We were able to assign relative potencies to each of the four regioisomers. In AVP controls basal $L_{\mathrm{p}}\left(\times 10^{-7} \mathrm{~cm} /\right.$ atm per s) increased from $5.86 \pm 1.6$ to a peak of $234.75 \pm 11.7(n=17)$ upon exposure of the tubules to $10 \mu \mathrm{U} / \mathrm{cm}^{2}$ vasopressin. Basal and peak $L_{\mathrm{p}}$ values were not different in tubules exposed to bath with or without vehicle $(0.001 \%$ ethanol) in either control or experimental protocols. Pretreatment of CCDs with $10^{-6} \mathrm{M} 11,12-$ DHET gave basal $L_{\mathrm{p}}$ measurements of $2.7 \pm 1.48, n=7$, NS from AVP controls. However, peak $L_{\mathrm{p}}$ in response to AVP in tubules pretreated with $1 \mu \mathrm{M} 11,12$-DHET was reduced to $178.56 \pm 7.7, n=7, P<0.005$ vs. AVP controls, a reduction in peak response of $25 \% .10^{-6} \mathrm{M} 5,6$-DHET resulted in a basal $L_{\mathrm{p}}$ value of $7.85 \pm 1.77, n=8$, again no different from control basal $L_{\mathrm{p}}$. The 5,6-DHET pretreatment blunted peak AVP-induced $L_{\mathrm{p}}$ by $35 \%$ to $154.35 \pm 11.9, n=8, P<0.005$. Tubules exposed to $10^{-6} \mathrm{M} 8,9$-DHET had basal $L_{\mathrm{p}}$ of $1.76 \pm 1.0$ and an AVP-induced peak $L_{\mathrm{p}}$ of $134.0 \pm 7.97, n=5, P<0.0001$, a suppression from the control response of $43 \%$. Finally, experiments using 14,15-DHET at $10^{-6} \mathrm{M}$ had a basal $L_{\mathrm{p}}$ of $4.20 \pm 1.3$ and a peak $L_{\mathrm{p}}$ of $95.2 \pm 8.39, n=5, P<0.0001$, a reduction of $60 \%$ from AVP controls. In these experiments we found that all four regioisomers of DHETs caused significant inhibition of the hydroosmotic effect of AVP in CCDs. 14,15DHET had the greatest effect on vasopressin-stimulated water transport.

Dose response of 14,15-DHET (Fig. 4). The inhibitory effect of 14,15-DHET on AVP-stimulated $L_{\mathrm{p}}$ is dose dependent and significant to nanomolar concentrations. Pretreatment with 14,15 -DHET at $10^{-6} \mathrm{M}$ reduced the AVP-peak $L_{\mathrm{p}}$ response to $95.2 \pm 8.39, n=5, P<0.0001$; at $10^{-7} \mathrm{M}$ this value was $110.0 \pm 7.22, n=5, P<0.0001$; at $10^{-8} \mathrm{M}$ the peak $L_{\mathrm{p}}$ was 


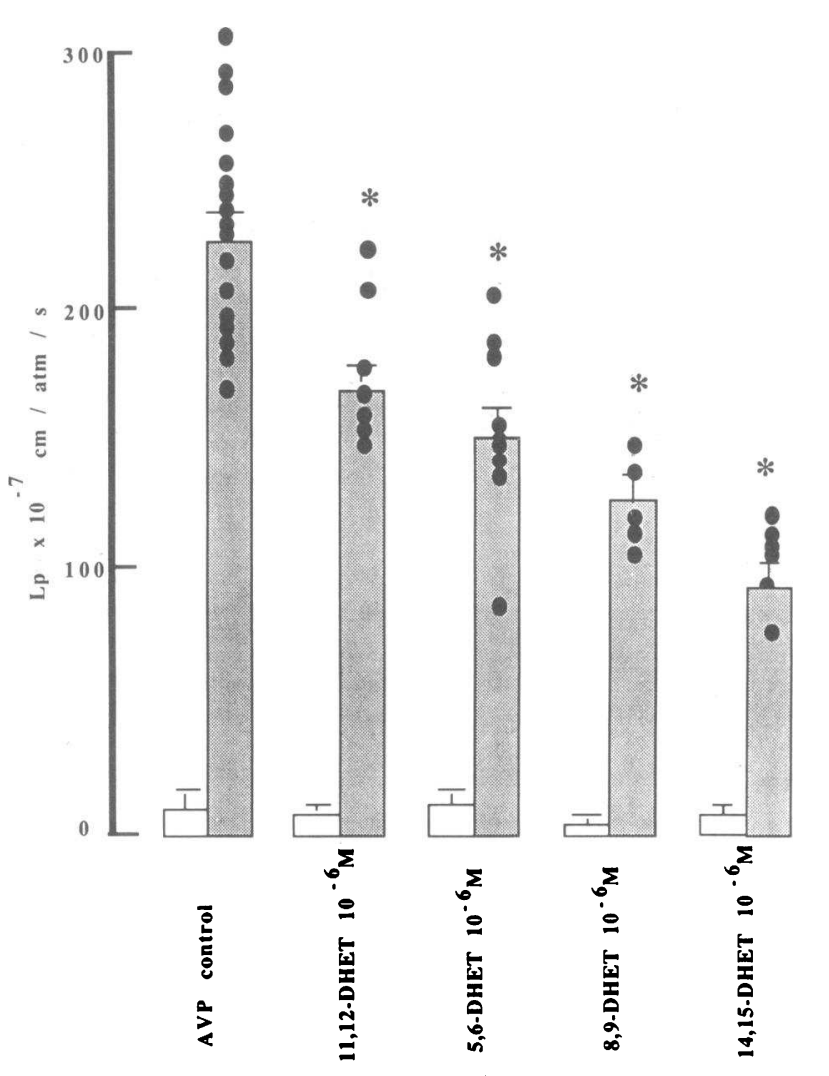

Figure 3. Effect of four regioisomeric DHETs on AVP-stimulated $L_{\mathrm{p}}$ in CCD. The diols are depicted in rank order of potency for inhibition of vasopressin-mediated water transport. Open bars, Basal $L_{\mathrm{p}}$; stippled bars, AVP-induced peak $L_{\mathrm{p}}$; dots, actual $L_{\mathrm{p}}$ values for individual experiments; *, significance of $P<0.0005$.

130.75 $\pm 1.88, n=4, P<0.0001$; at $10^{-9} \mathrm{M}$ peak $L_{\mathrm{p}}$ was 164.6 $\pm 10.61, n=5, P<0.005$. At concentrations of $10^{-10} \mathrm{M}$ of 14,15-DHET, the peak $L_{\mathrm{p}}$ was $235.0 \pm 4.93, n=3$, NS, a response no different from AVP controls.

Comparison of 14,15-DHET vs. $P G E_{2}$ on the AVP response (Fig. 5). We used previously cited concentrations of $\mathrm{PGE}_{2}$ used to inhibit AVP response in CCDs (26-28) to compare the inhibitory effect of 14,15 -DHET on this same process. 14,15DHET at $10^{-7} \mathrm{M}$ led to a $54 \%$ inhibition of AVP peak $L_{\mathrm{p}}$, 112.4 $\pm 9.94, n=5$, which was not statistically different from the effect produced by equimolar $\mathrm{PGE}_{2}(P=\mathrm{NS})$. $\mathrm{PGE}_{2}$ at $10^{-7} \mathrm{M}$ caused suppression of the AVP peak $L_{\mathrm{p}}$ to $88.8 \pm 13.4$, $n=5, P<0.0001$ when compared to AVP controls, a reduction of $63 \%$.

Effect of 14,15-DHET on CcAMP response (Fig. 6). CCDs were exposed to the permeant CAMP analogue CcAMP at $10^{-4}$ M. CcAMP is resistant to phosphodiesterase hydrolysis. CcAMP stimulated $L_{\mathrm{p}}$ to a peak $L_{\mathrm{p}}$ value of $189.6 \pm 11, n=8$. Pretreatment of tubules with $10^{-6} \mathrm{M} 14,15$-DHET reduced the peak $L_{\mathrm{p}}$ response to CcAMP to $132 \pm 13.4, n=5, P<0.01$, a reduction of $31 \%$ from control values. These experiments indicated that DHET was, at least in part, producing its inhibition of water flow in stimulated CCDs at a step beyond cAMP generation.

Analysis of harvested CCDs for époxygenase metabolites (Figs. 2, 7, and 8). The PFB ester derivatives isolated and purified from 550 microdissected CCDs were analyzed using capillary NICI GC/MS as described in Methods (Fig. 2). The

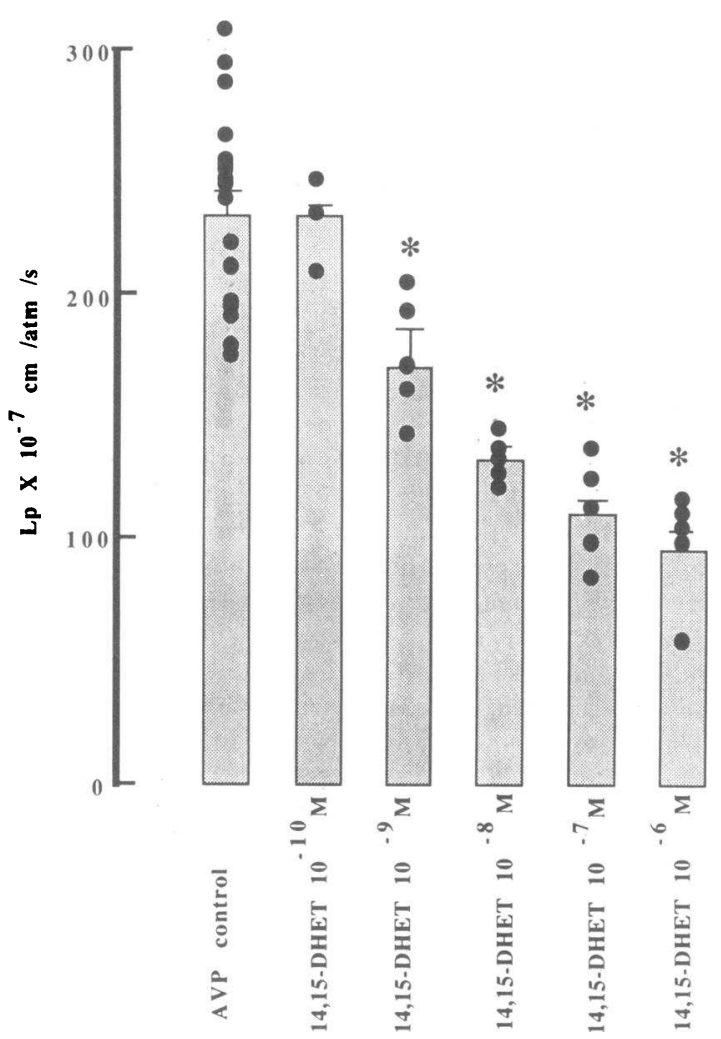

Figure 4. Dose response of 14,15-DHET on AVP-stimulated $L_{\mathrm{p}}$. Stippled bars, AVP-induced peak $L_{\mathrm{p}} ;$ dots, $L_{\mathrm{p}}$ values for each experiment; ${ }^{*}$, significance of $P<0.005$.

presence of EET mixtures in the biological samples was studied by comparisons of their GC and MS fragmentation properties to those of synthetic EET-PFBs. Under the conditions used, all four regioisomeric EETs coeluted from the GC column with a retention time $\left(R_{t}\right)$ of $\sim 11.8 \mathrm{~min}$. A summary of the experimental evidence suggesting the presence of EET mixtures in the rabbit CCDs is as follows: (a) NICI GC/mass

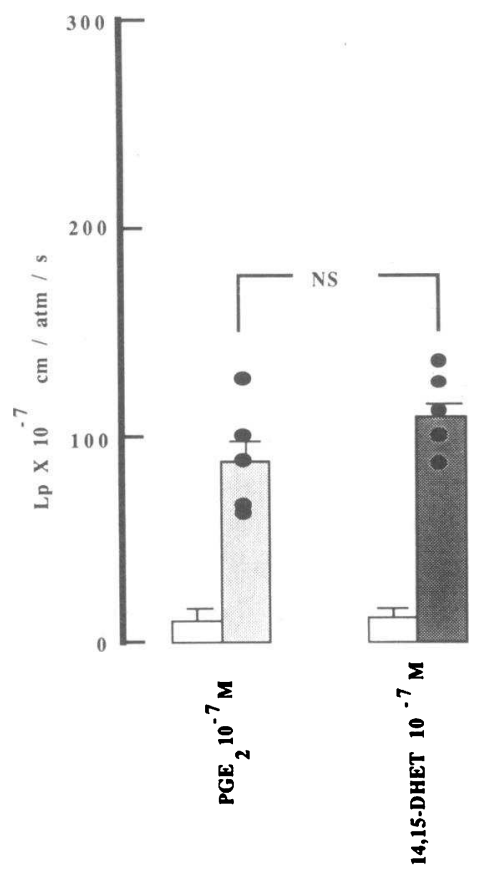

Figure 5. Inhibition of AVP-stimulated $L_{\mathrm{p}}$ by 14,15-DHET is as potent as $\mathrm{PGE}_{2}$. Open bars, Basal $L_{\mathrm{p}}$; stippled bars, experimental AVP-induced peak $L_{\mathrm{p}} ;$ dots, $L_{\mathrm{p}}$ values for each experiment. 


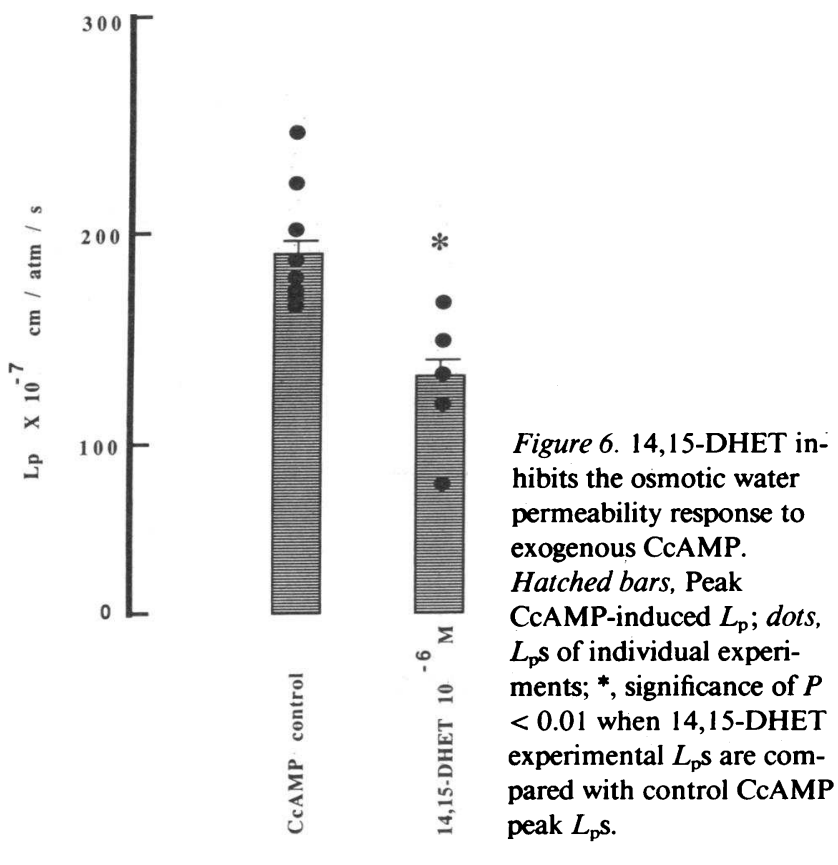

fragmentographic analysis of an aliquot of the PFB ester derivative of the biological sample shows coelution of the EETPFB-derived ion fragments at mass/ionic charge ratio $(\mathrm{m} / \mathrm{z})$ 319 (loss of PFB), 303 (loss of PFB and oxygen), and 301 (loss of PFB and water) with an $R_{\mathrm{t}}$ similar to that of an equimolar mixture of all four synthetic EET-PFBs (11.8 $\mathrm{min}$ ) as shown in Fig. $7 \mathrm{~A}$ ). The mass spectrum of the material present in the biological sample and elution from the GC column at 11.8 min shows the typical EET-PFB-derived ions at $m / z 319$ (base peak) and 303. With the exception of a contaminant ion fragment at $\mathrm{m} / z$ 311, the fragmentation pattern shown is almost identical to published EET-PFB spectra (Fig. $7 \mathrm{~B}$; 8). Coinjection of an aliquot of the biological material with a known quantity of synthetic $\left[5,6,8,9,11,12,14,15-{ }^{2} \mathrm{H}\right] 14,15$-EET-PFB shows the presence in the mixture of the EET-PFB-derived ion fragments at $m / z 319,303$, and 301 , and of an ion fragment at $m / z 327$, derived from the loss of PFB from the octadeuterated standard (Fig. $8 \mathrm{~B}$ ). The slight difference in retention times (6-7 s) between the protium and octadeuterated EET-PFB esters is due to well-established isotopic effects. The absence of contaminant ion fragments at $m / z 319,303$, and 301 in our synthetic $\left[{ }^{2} \mathrm{H}_{8}\right] 14,15-\mathrm{EET}$-PFB is illustrated by the mass spectrum of the material shown in Fig. 8 A.

\section{Discussion}

Oxygenated metabolites of AA are important modulators of cellular biochemistry and physiology. Much is known about cyclooxygenase metabolites. Less well examined are the arachidonate metabolites produced by lipoxygenases and cytochrome P450 NADPH-dependent epoxygenase. Since biologic activity has been demonstrated for cytochrome P450 epoxygenase metabolites of arachidonate in certain cells, we determined that the physiologic role they may fulfill in the kidney should be further studied.

The model system that we chose was a hormonally responsive epithelium, the rabbit $C C D$ perfused in vitro. The CCD is water impermeable under basal conditions but responds to vasopressin by becoming permeable to osmotically driven water flow. This hydroosmotic effect of AVP is accomplished at the intracellular level through generation of the second messenger, cAMP (26-30).

The present studies demonstrate a specific biologic action of all four regioisomers of DHETs. DHETs are potent inhibitors of the hydroosmotic effect of AVP in the mammalian CCD. However, equimolar concentrations of the separate isomers of DHET vary in the degree to which they suppress the response to AVP in this nephron segment. We find a rank order of increasing inhibitory effect as follows: 11,12-DHET $<$ 5,6-DHET < 8,9-DHET < 14,15-DHET (Fig. 3). 14,15DHET, the most potent epoxygenase-derived diol, blunts vasopressin's action in a dose-dependent fashion: by $60 \%$ at micromolar concentrations and $31 \%$ at nanomolar concentrations (Fig. 4).

The hydroosmotic effect of vasopressin in CCD is modulated by cyclooxygenase products, PGs of the E series (2, 4, 26-30). The current view is that PGs act at the level of a guanine nucleotide inhibitory regulatory protein that inhibits activation of adenylate cyclase and thereby inhibits generation of the second messenger cAMP (28-31). Vasopressin occupancy of its cell surface receptor causes a guanine nucleotide stimulatory regulatory protein to interact with the catalytic subunit of adenylate cyclase and promote endogenous cAMP formation. Exogenous CAMP or cAMP analogues like vasopressin stimulate osmotic water flow in CCDs (26-30). Exogenous or endogenous PGs inhibit the response to vasopressin in in vitro studies (27-30). At equimolar concentrations 14,15DHET caused suppression of AVP-stimulated water transport comparable to the suppression seen with $\mathrm{PGE}_{2}$ (Fig. 5). In contrast to studies reporting no effect of PGE on exogenous cAMP-mediated water flow, we found that DHET inhibited the permeability response to CcAMP (Fig. 6). 14,15-DHET was not as potent in suppressing water flow in response to CcAMP as it was in inhibiting the AVP response.

Our GC/MS data as well as previously published material strongly suggest the presence of epoxygenase products as endogenous constituents of rabbit kidney CCDs. The CCDs used for NICI GC/MS analysis were harvested with the same method used to isolate tubules for our microperfusion experiments. Similar techniques have been used to demonstrate the presence of 8,9-EET and 14,15-EET in whole rabbit kidneys (8) and to detect the presence of these same epoxides in human urine (24). Unfortunately, in the present case the limited quantity of material precluded a more conclusive analysis. While we have preliminary data to document endogenous EETs in CCDs, their specific location is unknown at present. However, in rat liver EETs are identified in phosphatidylinositol and phosphatidylethanolamine esterified to the $s n-2$ position of the glycerol backbone of these phospholipids (25). This finding may be important given that biologically active metabolites of arachidonate are classically felt to depend on metabolism of free AA generated through the action of phospholipase $A_{2}$ on cellular phospholipids (32). Biologically active epoxygenase products present in cell membrane phospholipids could be released by the action of either phospholipase $A_{2}$ or phospholipase C.

Schlondorff et al. recently reported studies concerning the effect of EETs and DHETs on vasopressin-induced water flow in amphibian urinary bladder, an epithelium that shares many of the transport functions of the mammalian collecting duct 


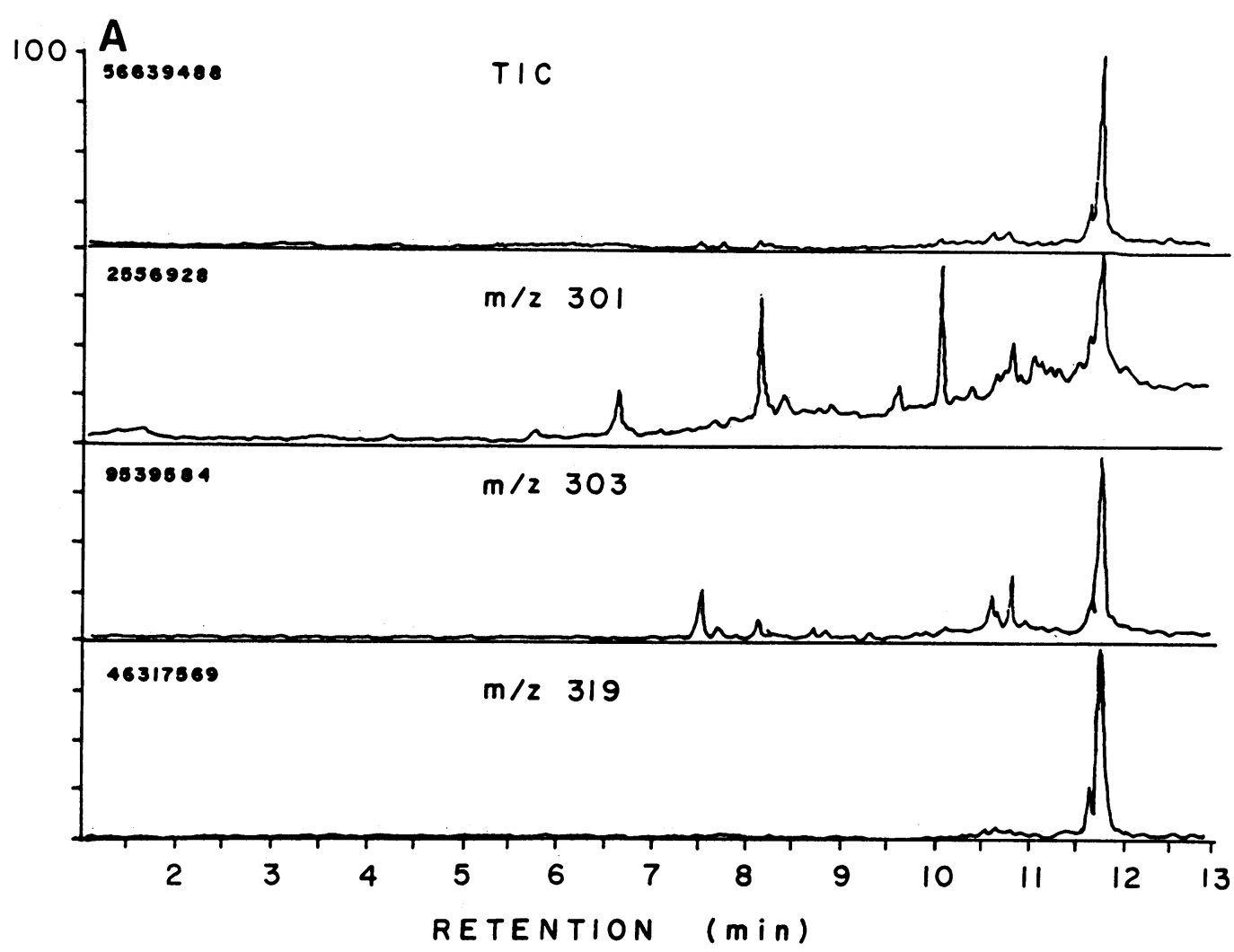

Figure 7. NICI GC/MS analysis of the sample extracted and purified from rabbit kidney $C C D$. An aliquot of the PFB derivative of the biological sample was injected into a 30-m SPB-5 fused silica capillary column. Column eluents were monitored using NICI MS as described in Methods. $A$, Mass fragmentographic analysis of the GC column eluent performed by selective ion monitoring at $m / z 319$ (loss of PFBO), 303 (loss of PFB and oxygen), and 301 (loss of PFB and water). Abscissa, $R_{\mathrm{t}}$ in minutes; ordinate, percent abundance. $B$, Mass spectrum of the material eluting with a GC retention time similar to that of synthetic 14,15-EET-PFB (11 min, $46 \mathrm{~s}$, or 11.8 min). Abscissa, $m / z$; ordi$m / z$

(18). Their study also reports significant inhibitory activity for EETs and DHETs on AVP-mediated water transport. Experiments were performed at room temperature using mounted toad hemibladders. 11,12-EET and 5,6-EET and their respective diols (DHETs) produced inhibition of the vasopressin response. However, to achieve significance, concentrations of $10^{-6}-10^{-5} \mathrm{M}$ of these compounds were required. EETs and DHETs also inhibited vasopressin-induced adenylate cyclase activity. The inhibitory effect on AVP's action by 11,12-EET was comparable to that seen with $\mathrm{PGE}_{2}$. 11,12-EET, as op- posed to the other isomers, also reduced osmotic water flow in response to 8-bromo-3',5' cAMP. These authors proposed a mechanism of action for EETs and DHETs on AVP activity through an inhibition of AVP-induced cAMP generation (18). Experiments presented in this communication differ importantly from the studies of Schlondorff et al. in the following ways: (a) our experiments were performed in a mammalian system at $37^{\circ} \mathrm{C} ;(b)$ the concentrations of eicosanoid used were more likely to be physiologically relevant (the concentration of EETs in normal, pooled human urine was $\sim 2 \mathrm{nM}$; see refer- 


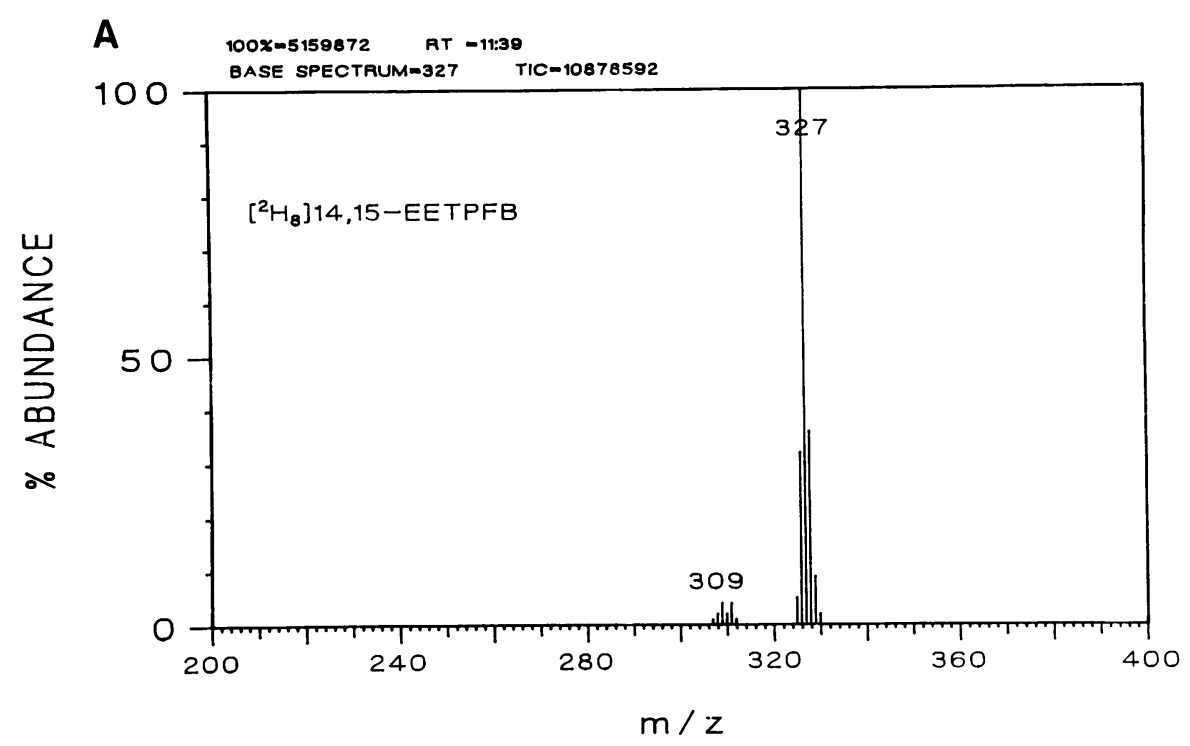

B
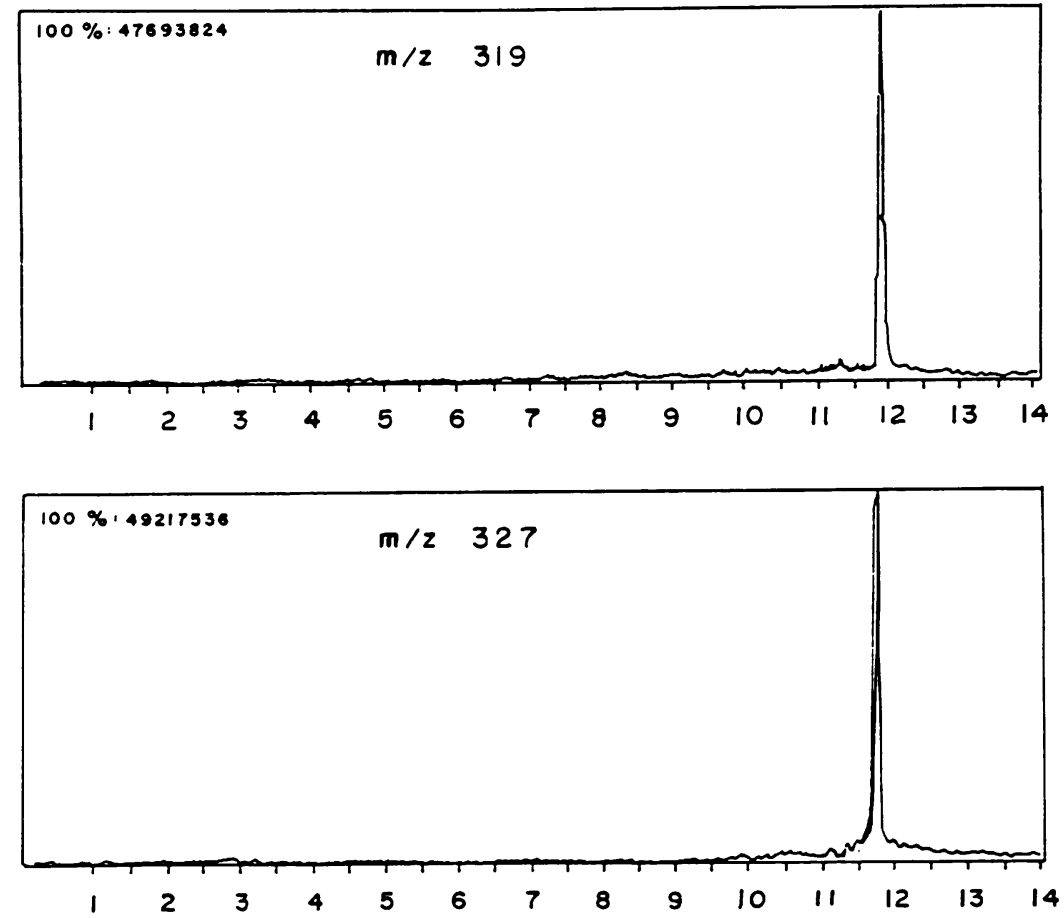

Figure 8. NICI GC/MS analysis of a coinjected mixture of the sample extracted and purified from rabbit kidney $C C D$ and an aliquot of synthetic $\left[{ }^{2} \mathrm{H}_{8}\right] 14,15$-EET. $A, \mathrm{~A}$ 5-ng sample of synthetic $\left[{ }^{2} \mathrm{H}_{8}\right] 14,15$-EETPFB ester was injected into the capillary GC column and the eluent monitored by NICI MS. Shown is the mass spectrum of the material eluting from the GC column at $\sim 11 \mathrm{~min}, 39 \mathrm{~s}$ or $11.65 \mathrm{~min}$. Abscissa, $m / z$; ordinate, percent abundance. $B$, An aliquot of the biological sample was mixed with a known quantity of synthetic $\left[{ }^{2} \mathrm{H}_{8}\right]-$ 14,15-EET-PFB ( $1 \mathrm{ng})$ and then injected into the capillary GC column. The column eluent was monitored by NICI mass fragmentography at $m / z 319$ and 327 . Selected ion current profile under NICI conditions for the EET-PFB ester present in the biological sample, $m / z 319$ (M-PFB); and $\left[{ }^{2} \mathrm{H}_{8}\right] 14,15$-EET-PFB ester, $m / z 327$ (MPFB).

ence 24); and (c) our data indicating that DHET inhibits the osmotic water flow induced by exogenous cAMP suggests that epoxygenase metabolites may influence water transport through some process other than, or in addition to, reduction of adenylate cyclase activity.

Determination of the mechanism whereby cytochrome P450 metabolites of arachidonate inhibit water transport in CCD will require future experiments. To address any component of DHET action that involves cAMP generation will require measurements of cAMP production. It has been shown that calcium and calcium-activated, phospholipid-dependent protein kinase $\mathrm{C}$ inhibit the hydroosmotic effect of vasopressin in CCD and amphibian urinary bladder (33-36). Importantly, Ando et al. (33) demonstrated that protein kinase $C$ activation leads to an inhibition of vasopressin-mediated water transport through a post-cAMP process. Thus it is possible that the DHETs transduce their inhibitory effect through changes in cell calcium or activation of protein kinase C. Support for this notion is derived from studies demonstrating that EETs affect calcium binding, uptake, and release from microsomes made from vascular smooth muscle (35). This hypothesis remains to be tested.

In summary, we have presented evidence that DHETs produced by cytochrome P450 NADPH-dependent epoxygenase are potent inhibitors of the hydroosmotic effect of vasopressin in rabbit CCD. The 14,15-DHET isomer evokes the most potent suppressive action, and the inhibition is dose dependent. DHET is as potent as $\mathrm{PGE}_{2}$ in inhibiting AVP-mediated water transport, a process thought to be mediated via cAMP. We also have shown that these P450 metabolites of AA are present in the normal CCD. Finally, although the specific mechanism(s) by which these compounds produce their inhibitory effect will require further study, their inhibition of vasopressin action in the CCD involves a post-cAMP step. 


\section{Acknowledgments}

We thank Ms. Deborah Asbell-Gillespie for her technical assistance.

This study was supported by grant DK-39261-02 from the National Institutes of Health, National Institute of Diabetes and Digestive and Kidney Diseases. Dr. Hirt was a fellow of the National Kidney Foundation (NKF) and NKF-Middle Tennessee Chapter.

\section{References}

1. Jackson, E. K., R. A. Branch, H. S. Margolis, and J. A. Oates. 1985. Physiologic functions of the renal prostaglandin, renin, and kallikrein systems. In The Kidney: Physiology and Pathophysiology. D. W. Seldin and G. Geibisch, editors. Raven Press, New York. 613-644.

2. Schlondorff, D., and R. Ardaillou. 1986. Prostaglandins and other arachidonic acid metabolites in the kidney. Kidney Int. 29:108119.

3. Currie, M. G., and P. Needleman. 1984. Renal arachidonic acid metabolism. Annu. Rev. Physiol. 46:327-341.

4. Bonvalet, J.-P., P. Pradilles, and N. Farman. 1987. Segmental synthesis and actions of prostaglandins along the nephron. Am. J. Physiol. 253:F377-F387.

5. Morrison, A. R., and N. Pascoe. 1981. Metabolism of arachidonic acid through NADPH-dependent oxygenase of renal cortex. Proc. Natl. Acad. Sci. USA. 79:767-770.

6. Capdevila, J., L. J. Marnett, N. Chacos, R. A. Prough, and R. Estabrook. 1982. Cytochrome P450 dependent oxygenation of arachidonic acid to hydroxyeicosatetranoic acids. Proc. Natl. Acad. Sci. USA. 79:767-770.

7. Oliw, E. H., F. P. Guengerich, and J. A. Oates. 1982. Oxygenation of arachidonic acid by hepatic monoxygenases. J. Biol. Chem. 257:3771-3781.

8. Falck, J. R., V. J. Schueler, H. R. Jacobson, A. K. Siddhanta, B. Pramanik, and J. Capdevila. 1987. Arachidonate epoxygenase: identification of epoxyeicosatreinoic acids in rabbit kidney. J. Lipid Res. 28:840-846.

9. Capdevila, J., N. Chacos, J. Werringloer, R. A. Prough, and R. W. Estabrook. 1981. Liver microsomal cytochrome P-450 and the oxidative metabolism of arachidonic acid. Proc. Natl. Acad. Sci. USA. 78:5362-5366.

10. Fitzpatrick, F. A., and R. C. Murphy. 1988. Cytochrome P450 metabolism of arachidonic acid: formation and biological actions of "epoxygenase"-derived eicosanoids. Pharmacol. Rev. 40:229-241.

11. Jacobson, H. R., S. Corona, J. Capdevila, N. Chacos, S. Manna, A. Womack, and J. R. Falck. 1985. Effects of epoxyeicosatrienoic acids on ion transport in the rabbit cortical collecting tubule. In Prostaglandins and Membrane Ion Transport. P. Braquet, R. P. Garay, J. C. Frolich, and S. Nicosia, editors. Raven Press, New York. 311-318.

12. Schwartzman, M., N. R. Ferreri, M. A. Carroll, E. Songu-Mize, and J. McGiff. 1985. Renal cytochrome P450-related arachidonate metabolite inhibits ( $\mathrm{Na}+\mathrm{K}$ ) ATPase. Nature (Lond.). 314:620-622.

13. McGiff, J., and M. A. Carroll. 1987. Cytochrome P-450-related arachidonic acid metabolites. Am. Rev. Respir. Dis. 136:488-491.

14. Fitzpatrick, F. A., M. D. Ennis, M. E. Baze, M. A. Wynalda, J. E. McGee, and W. F. Liggett. 1986. Inhibition of cyclooxygenase activity and platelet aggregation by epoxyeicosatrienoic acids. J. Biol. Chem. 261:15334-15338.

15. Carroll, M. A., M. Schwartzman, J. Capdevila, J. R. Falck, and J. McGiff. 1987. Vasoactivity of arachidonic acid epoxides. Eur. $J$. Pharmacol. 138:281-283.

16. Schwartzman, M., M. A. Carroll, N. G. Ibraham, N. R. Ferreri, E. Songu-Mize, and J. C. McGiff. 1985. Renal arachidonic acid metabolism: the third pathway. Hypertension (Dallas). 7(Suppl I):I-135-I144.

17. Negro-Vilar, A., G. D. Snyder, J. R. Falck, S. Manna, N.
Chacos, and J. Capdevila. 1985. Involvement of eicosanoids in release of oxytocin and vasopressin from the neural lobe of the rat pituitary. Endocrinology. 116:2663-2668.

18. Schlondorff, D., E. Petty, J. A. Oates, M. Jacoby, and S. D. Levine. 1987. Epoxygenase metabolites of arachidonic acid inhibit vasopressin response in toad bladder. Am. J. Physiol. 253:F464-F470.

19. Burg, M., J. Grantham, M. Abramow, and J. Orloff. 1966. Preparation and study of fragments of single rabbit nephrons. Am. J. Physiol. 210:1293-1298.

20. Corey, E. J., A. Marfat, J. R. Falck, and J. O. Albright. 1980. Controlled chemical synthesis of the enzymatically produced eicosanoids 11-, 12- and 15-HETE from arachidonic acid and conversion to the corresponding hydroperoxides (HPETE). J. Am. Chem. Soc. 102:1433-1435.

21. Falck, J. R., and S. Manna. 1982. 8,9-Epoxyarachidonic acid: a cytochrome P-450 metabolite. Tetrahedron Lett. 23:1755-1756.

22. Corey, E. J., H. Niwa, and J. R. Falck. 1979. Selective epoxidation of eicosa-cis-5,8,11,14-tetraenoic (arachidonic) acid and eicosacis-8,11,14-trienoic acid. J. Am. Chem. Soc. 101:1586-1587.

23. Du Bois, R., A. Veniory, and M. Abramow. 1976. Computation of the osmotic water permeability of perfused tubule segments. Kidney Int. 10:478-479.

24. Toto, R., A. Siddhanta, S. Manna, B. Pramanik, J. R. Falck, and J. Capdevila. 1987. Arachidonic acid epoxygenase: detection of epoxyeicosatreinoic acids in human urine. Biochim. Biophys. Acta. 919:132-139.

25. Capdevila, J., V. Kishore, E. Dishman, and I. A. Blair. 1987. A novel pool of rat liver inositol and ethanolamine phospholipids contains epoxyeicosatrienoic acids (EETs). Biochem. Biophys. Res. Commun. 146:638-644.

26. Torikai, S., and K. Kurokawa. 1983. Effect of $\mathrm{PGE}_{2}$ on vasopressin-dependent cell cAMP in isolated single nephron segments. Am. J. Physiol. 245:F58-F66.

27. Grantham, J. J. and J. Orloff. 1968. Effect of prostaglandin $E_{1}$ on the permeability response of the isolated collecting tabule to vasopressin, adenosine $3^{\prime}, 5^{\prime}$-monophosphate and theophylline. J. Clin. Invest. 47:1154-1161.

28. Nadler, S. P., S. C. Hebert, and B. M. Brenner. 1986. $\mathrm{PGE}_{2}$, forskolin and cholera toxin interactions in rabbit cortical collecting tubule. Am. J. Physiol. 250:F127-F135.

29. Abramow, M., R. Beauwens, and E. Cogan. 1987. Cellular events in vasopressin action. Kidney Int. 32(Suppl 21):S-56-S-66.

30. Schuster, V. I. 1985. Mechanism of bradykinin, ADH and cAMP interaction in rabbit cortical collecting duct. Am. J. Physiol. 249:F645-F653.

31. Watanabe, T., K. Umegaki, and W. L. Smith. 1986. Association of a solubilized prostaglandin $E_{2}$ receptor from renal medulla with a pertussis toxin-reactive guanine nucleotide regulatory protein. $J$. Biol. Chem. 261:13430-13439.

32. Vanden Bosh, H. 1980. Intracellular phospholipase A. Biochim. Biophys. Acta. 604:191-246.

33. Ando, Y., H. R. Jacobson, and M. D. Breyer. 1987. Phorbol myristate acetate, dioctanoylglycerol and phosphatidic acid inhibit the hydroosmotic effect of vasopressin on rabbit cortical collecting tubule. J. Clin. Invest. 80:590-593.

34. Schlondorff, D., and D. Levine. 1985. Inhibition of vasopressin-stimulated water flow in toad bladder by phorbol myristate acetate, dioctanoylglycerol, and RHC-80267. J. Clin. Invest. 76:1071-1078.

35. Kutsky, P., J. Falck, G. Weiss, S. Manna, N. Chacos, and J. Capdevila. 1983. Effects of newly reported arachidonic acid metabolites on microsomal $\mathrm{Ca}^{++}$binding, uptake and release. Prostaglandins. 26:13-21.

36. Teitelbaum, I., and T. Berl. 1986. Effects of calcium on vasopressin-mediated cyclic adenosine monophosphate formation in cultured rat inner medullary collecting tubule cells. J. Clin. Invest. 77:1574-1583. 